\title{
Evolution of dispersal under a fecundity-dispersal trade-off
}

\section{Weigang, Helene C.}

2015-04-21

Weigang , H C \& Kisdi , E 2015 , ' Evolution of dispersal under a fecundity-dispersal trade-off

' , Journal of Theoretical Biology , vol. 371 , pp. 145-153 . https://doi.org/10.1016/j.jtbi.2015.02.013

http://hdl.handle.net/10138/262547

https://doi.org/10.1016/j.jtbi.2015.02.013

acceptedVersion

Downloaded from Helda, University of Helsinki institutional repository.

This is an electronic reprint of the original article.

This reprint may differ from the original in pagination and typographic detail.

Please cite the original version. 


\title{
Evolution of dispersal under a fecundity-dispersal trade- off
}

\author{
Helene C. Weigang ${ }^{a *}$ \\ Eva Kisdi ${ }^{b}$ \\ Department of Mathematics and Statistics \\ University of Helsinki \\ PO Box 68, FIN-00014 Finland \\ email: ${ }^{a}$ helene.weigang@helsinki.fi ${ }^{b}$ eva.kisdi@helsinki.fi \\ *corresponding author
}

This article has been published in the Journal of Theoretical Biology:

http://dx.doi.org/10.1016/j.jtbi.2015.02.013

Please cite as:

Weigang H. C. \& É. Kisdi. 2015. Evolution of dispersal under a fecundity-dispersal trade-off. J. theor. Biol. 371: 145153.

Creative Commons CC BY NC ND license

Keywords:Adaptive dynamics, critical function analysis, evolutionary branching, life history, emigration

\begin{abstract}
Resources invested in dispersal structures as well as time and energy spent during transfer may often decrease fecundity. Here we analyse an extended version of the Hamilton-May model of dispersal evolution, where we include a fecundity-dispersal trade-off and also mortality between competition and reproduction. With adaptive dynamics and critical function analysis we investigate the evolution of dispersal strategies and ask whether adaptive diversification is possible. We exclude evolutionary branching for concave trade-offs and show that for convex trade-offs diversification is promoted in a narrow parameter range. We provide theoretical evidence that dispersal strategies can monotonically decrease with increasing survival during dispersal. Moreover, we illustrate the existence of two alternative attracting dispersal strategies. The model exhibits fold bifurcation points where slight changes in survival can lead to evolutionary catastrophes.
\end{abstract}




\section{Introduction}

Dispersal is an elementary driver of ecology and evolution, which fundamentally shapes the distribution, abundance and diversity of species, and plays a key role in their persistence in the face of habitat fragmentation, climate change, and other adverse environmental impacts. Dispersal helps to avoid crowding, kin competition and inbreeding, and to escape from natural enemies. However, dispersal has multifarious costs (reviewed by Bonte et al., 2012; Travis et al., 2012): Investments into the ability to disperse (e.g., into flight muscles) imply energetic costs that are paid prior to dispersal and often concern all individuals, also those who eventually do not disperse. During transfer, there is typically a high risk of mortality and a risk of not arriving in any suitable habitat, which affects the individuals who do disperse. The transfer process itself has extra energetic costs, which may impinge on the condition of the individual, and it also needs time, whereby priority advantages may be lost. Upon settlement, immigrants may be affected by various costs related to loss of familiarity and social rank. Many of these costs depend also on the distance traversed during dispersal.

Despite the great variety and rich biological context of dispersal costs, most models consider only a fixed mortality cost (combining mortality during transfer and the risk of landing in an unsuitable habitat), a fixed cost for each dispersal distance (e.g., Rousset and Gandon, 2002), or a fixed mortality cost per movement step (e.g., Bonte et al., 2010; Poethke et al., 2011). Fixed mortality costs generate rigid trade-offs between the number of non-dispersed and successfully dispersed offspring. In reality, however, organisms have evolved many ways to alleviate mortality during dispersal; but this is unavoidably costly and induces trade-offs elsewhere. For example, investing resources into better sensory or flight capabilities reduces mortality during dispersal (Bonte et al., 2012; Travis et al., 2012), but the resources used to this end represent an energetic cost of dispersal.

More specifically, energetic costs of dispersal (e.g., investment into morphological structures such as flight muscles or energy spent during transfer) can easily enforce a trade-off with fecundity. Indeed, there is good empirical evidence for fecundity costs of dispersal ability in insects (Roff, 1977), especially in wing-dimorphic species (Mole and Zera, 1993; Roff, 1984; Stirling et al., 2001; Wratten, 1977; Zera, 1984; reviewed by Roff, 1986; Zera and Denno, 1997) and in Lepidoptera (Gu and Danthanarayana, 1992; Gu et al., 2006; Hughes et al., 2003; Karlsson and Johansson, 2008; but see Hanski et al., 2006). In plants, seed dispersal depends on plant height more than on seed size (Thomson et al., 2011), and investments into tall stems may cause a trade-off between dispersal ability and seed number when dispersal is facilitated by wind. Furthermore, dispersal may occur via the production of costly fruits, which also leads to a trade-off between dispersal and fecundity.

When fecundity is antagonistically interacting with dispersal, it is unclear what shape the trade-off function should be. Energy allocated to dispersal structures can be measured directly in terms of lost fecundity (e.g., the number of eggs energetically equivalent to the dispersal structure). The probability of dispersal is likely an increasing function of the energy allocated to dispersal structures (Roff and Fairbairn, 1991), but the exact shape of this function is unclear. Existing models assume some simple function without sufficient justification (e.g., Burton et al., 2010; King and Roff, 2010; but see Cohen and Motro, 1989). Other models assume that investment into dispersal-related traits reduces the mortality risk during transfer (Fronhofer et al., 2011; Travis et al., 2012). This improves the classic assumption of a fixed mortality cost, yet 
the function linking investment and risk is chosen only for its simplicity. Similar choices are made when seed dispersal is linked to seed size (Ezoe, 1998; Levin and Muller-Landau, 2000). These choices of functions amount to assuming ad hoc trade-offs between fecundity and (successful) dispersal. Importantly, seemingly minor details of the trade-off functions can change the qualitative predictions of a model (de Mazancourt and Dieckmann, 2004; Geritz et al., 2007). Hence assuming an arbitrary trade-off function, even if it is a biologically plausible function, can unjustifiably distort the results.

In this paper, we investigate the adaptive dynamics of dispersal (i.e., emigration) under a dispersal-fecundity trade-off. In Section 2, we include the trade-off in a generalised version of the model of Hamilton and May (1977) where some individuals may die before reproduction. In order to avoid ad hoc choices of trade-off functions, in Section 3 we analyse the model without committing to any particular trade-off shape.

In Section 3.1, we employ the technique of critical function analysis (de Mazancourt and Dieckmann, 2004; Kisdi, 2006, 2014). In critical function analysis, we identify the conditions under which various evolutionary outcomes are possible in terms of the local properties (such as the slope and the curvature) of the unspecified trade-off function. This technique differs from a traditional bifurcation analysis in treating the slope and the curvature of the trade-off function directly as bifurcation parameters, instead of first fixing a parameterised trade-off function and then analysing the model in terms of the parameters. In the present model, we show that all generic types of evolutionary singularities can occur, provided that there is some mortality between establishment and reproduction. In particular, dispersal may evolve to an evolutionarily stable level, may undergo evolutionary branching, or there may be a repelling singularity leading to multiple attractors of dispersal evolution. For comparison, we analyse the model also without the dispersal-fecundity trade-off (i.e., assuming constant fecundity; see Appendix A) and show that in this case, no evolutionary branching and no multiple attractors can occur.

In Section 3.2, we investigate how the evolutionary singularity changes with survival before reproduction and with survival during dispersal. For the latter, we find a novel pattern of dispersal evolution demonstrated in the example of Section 4: dispersal can monotonically decrease with increasing survival during dispersal over its entire range, or else (as found earlier, see Discussion) it may also increase or vary non-monotonically. In the example of Section 4, we also illustrate evolutionary branching, show an example for the co-evolution of divergent dispersal strategies after evolutionary branching, present examples for multiple attractors, and highlight the possibility of evolutionary catastrophes due to slight changes in survival probabilities at fold bifurcation points.

\section{Model}

Consider a semelparous population with non-overlapping generations that reproduces asexually and lives in a fragmented landscape. After competition between juveniles, each site is occupied by one individual that survives until reproduction with probability $s>0$. We refer to $s$ as adult survival (but note that this precedes the only time when the individual reproduces). At the time of reproduction a fraction $1-s$ of sites is empty. All individuals who survived to reproduction give birth to $B\left(x_{i}\right)$ offspring and die. The offspring disperse with probability $x_{i}$, join a global dispersal pool and survive dispersal with probability $p>0$. The dispersers land on an empty site 
with probability $1-s$, or on an occupied site with probability $s$, where non-dispersed offspring are present (i.e. higher competition). After dispersal, one individual establishes at each site (fair competition) and survives until reproduction with probability $s$.

The production of offspring with dispersal morphologies is costly. Since resources are limited and energy finite, individuals who invest more into the dispersal apparatus of their offspring produce fewer offspring than individuals who invest little into dispersal structures. Hence, the number of offspring of the latter individuals is high compared to the former, but comes at a cost of reduced dispersal probability, which increases kin competition and decreases the chances of colonising new sites. We include such a fecundity-dispersal trade-off in the form $B\left(x_{i}\right)=\kappa b\left(x_{i}\right)$, where $b\left(x_{i}\right)$ describes the trade-off between the dispersal probability and fecundity. The function $b\left(x_{i}\right)$ is non-negative and decreasing for all $x_{i} \in[0,1]$.

Let $n_{i}(t)$ be the fraction of $M$ sites that adults with dispersal trait $x_{i}$ occupy at time $t$. Assuming that both the number of individuals born in every patch and the number of patches is large $(M, \kappa \rightarrow \infty)$, we can neglect stochastic effects. We then obtain $n_{i}(t+1)$ as the number of offspring produced $\left(n_{i}(t) s b\left(x_{i}\right)\right)$ times the probability that the offspring wins a site. Offspring compete for sites in three ways: First, an offspring stays in the natal site and competes for the natal site; second, the offspring successfully disperses (i.e., emigrates and survives dispersal) and lands in a site where offspring have been born; and third, the offspring successfully disperses and lands in a site that was empty before immigration. In each case, the probability of winning the site is one over the number of competitors, but the number of competitors varies in different sites. This leads to the three terms of the discrete-time population model

$$
n_{i}(t+1)=n_{i}(t) s b\left(x_{i}\right)\left(\frac{\left(1-x_{i}\right)}{s p E_{1}+\left(1-x_{i}\right) b\left(x_{i}\right)}+s x_{i} p E_{2}+\frac{(1-s) x_{i} p}{s p E_{1}}\right),
$$

where $E_{1}$ and $E_{2}$ are the environmental feedback variables given by $E_{1}=\sum_{j=1}^{k} n_{j}(t) b\left(x_{j}\right) x_{j}$ and $E_{2}=\sum_{l=1}^{k} \frac{n_{l}(t)}{\left(1-x_{l}\right) b\left(x_{l}\right)+s p E_{1}}$ and $k$ is the number of dispersal strategies. $E_{1}$ is the average number of individuals that disperse from a site, and $E_{2}$ gives the probability that a disperser establishes upon landing at an occupied site. Since feedback variables, of which we have two, set an upper limit to the number of coexisting strategies (Geritz et al., 1997) we conclude that at most two dispersal strategies coexist.

This model is an extension of the Hamilton-May model (Hamilton and May, 1977) with a trade-off between dispersal and fecundity (Cohen and Motro, 1989) and the possibility of death before reproduction.

\section{Adaptive dynamics of dispersal}

To determine the long-term evolution of dispersal we investigate a series of invasion-fixation events. We assume that $(i)$ the ecological time-scale is faster than the evolutionary, (ii) mutations have small effects on the evolving dispersal trait, and (iii) the mutant population is initially small compared to the resident population. These assumptions allow us to use the adaptive dynamics toolbox (Geritz et al., 1998). The invasion fitness of a mutant population with trait $y$ into an environment inhabited by a resident population with trait $x$ is derived from 
Eq. (1) for $k=2$ and given by the following function:

$$
w(x, y)=s b(y)\left(\frac{(1-y)}{b(x) x p s+b(y)(1-y)}+\frac{y p s}{b(x) x p s+b(x)(1-x)}+\frac{y p(1-s)}{b(x) x p s}\right) .
$$

Directional selection ceases at the singular strategy $x^{*}$, where $D\left(x^{*}\right)=\partial w /\left.\partial y\right|_{y=x=x^{*}}=0$. This is equivalent to:

$$
b^{\prime}\left(x^{*}\right)=\frac{b\left(x^{*}\right)}{x^{*}}\left[\frac{p s^{2} x^{*}}{(1-s)\left(1-x^{*}\right)^{2}+p^{2} s^{2} x^{* 2}+2 p s\left(1-x^{*}\right) x^{*}}-1\right] .
$$

Note that when $s \rightarrow 0$ or $p \rightarrow 0$ (with $s M \rightarrow \infty$ and $s p \kappa \rightarrow \infty$ ), the singular dispersal strategy is the solution to

$$
b^{\prime}\left(x^{*}\right)=-\frac{b\left(x^{*}\right)}{x^{*}}
$$

In this limit, the number of immigrants arriving at the natal site becomes negligible and competition vanishes. In this case, any non-dispersed offspring can retain the natal site and the favoured strategy, i.e., the singular strategy, maximises the number of dispersers $x b(x)$.

A singular strategy is evolutionarily stable if

$$
E=\left.\frac{\partial^{2} w(x, y)}{\partial y^{2}}\right|_{x=y=x^{*}}<0
$$

and attracting (convergence stable) if

$$
E+M=\frac{\partial^{2} w(x, y)}{\partial y^{2}}+\left.\frac{\partial^{2} w(x, y)}{\partial x \partial y}\right|_{x=y=x^{*}}<0
$$

(Christiansen, 1991; Eshel, 1983). In Appendix A we analyse the evolutionary dynamics without a trade-off (i.e., assuming that $b$ is constant). In this case, the model always has a unique singular strategy that is both attracting and evolutionarily stable, i.e., a $C S S$.

\subsection{Critical function analysis}

Here we use critical function analysis (de Mazancourt and Dieckmann, 2004; Kisdi, 2006, 2014) to analyse evolutionary scenarios without pre-defining a specific trade-off shape. Let $b_{\text {crit }}(x)$ be a solution of the differential equation in (3) (note that there are infinitely many solutions with different initial values). The function $b_{\text {crit }}(x)$ is called the critical function and any point along the critical function can be made singular by choosing a trade-off tangential to the critical function at that point.

We obtain the critical function analytically with Mathematica (Wolfram Research, version 8.0.4.0):

$$
b_{\text {crit }}(x)=\frac{b_{0} x_{0}}{x} \exp \left(f\left(x_{0}\right)-f(x)\right)
$$


for the initial condition $b\left(x_{0}\right)=b_{0}$, where

$$
f(x)=\sqrt{s} \operatorname{ArcTanh}\left(\frac{x\left[(1-p s)^{2}-s\right]-[(1-p s)-s]}{p \sqrt{s^{3}}}\right) .
$$

If the argument of the ArcTanh lies outside of $(-1,1)$, the function value is complex. For some parameter values of $s$ and $p$ the argument can be smaller than -1 for all $x$ (see Appendix B.1). Then the imaginary part of $f(x)$ cancels with the imaginary part of $f\left(x_{0}\right)$ and the critical function remains real (see Appendix B.1). In Appendix B.2 we show that the critical function is convex. Singular strategies of trade-offs with local curvature smaller than $b_{c r i t}^{\prime \prime}(x)$ are attracting, and repelling if the opposite holds (de Mazancourt and Dieckmann, 2004).

Additionally, we determine the critical curvature for evolutionary stability. Since $E$ (see inequality (4)) depends on the curvature of the trade-off function, we rewrite $E$ as $b^{\prime \prime}\left(x^{*}\right)-e\left(x^{*}\right)$, where $x^{*}$ is the point of tangent between the trade-off and a critical function. A singular strategy is an $E S S$ if $E<0$, i.e. the curvature of the trade-off function is smaller than the critical curvature of evolutionary stability $e\left(x^{*}\right)$. In Appendix B.3 we prove that $e\left(x^{*}\right)$ is positive.

The second term of inequality (5), $M$, is independent of the trade-off curvature but depends on the slope of the trade-off at $x^{*}$, which is determined by the slope of the critical function. If $M$ is negative, evolutionary branching is possible by choosing the trade-off curvature such that $E$ is positive but $E+M$ is negative, i.e., the singularity is an attractor (inequality (5) holds) but not evolutionary stable (the opposite of (4) holds; see details below). We find that $M$ is negative if

$$
\frac{p^{2} x^{* 2}}{\left(1-x^{*}\right)^{2}}<\frac{1-s}{s^{2}}
$$

and positive if the opposite holds. Note that for $s=1$, inequality (6) is never fulfilled and evolutionary branching is impossible (see Hamilton and May, 1977). Since $M$ can be of both signs depending on the parameters $s, p$ and on $x^{*}$, the point of tangent between the trade-off and a critical function (which can also be chosen freely by choosing an appropriate trade-off), it is possible to construct trade-offs that lead to any type of singularity. If $x^{*}$ is chosen such that inequality (6) holds, then $e\left(x^{*}\right)<b_{c r i t}^{\prime \prime}\left(x^{*}\right)$, and hence trade-offs with an intermediate curvature, i.e., with $e\left(x^{*}\right)<b^{\prime \prime}\left(x^{*}\right)<b_{\text {crit }}^{\prime \prime}\left(x^{*}\right)$, lead to evolutionary branching, whereas trade-offs with $b^{\prime \prime}\left(x^{*}\right)<e\left(x^{*}\right)$ yield an attracting evolutionary stable strategy and trade-offs with $b^{\prime \prime}\left(x^{*}\right)>$ $b_{c r i t}^{\prime \prime}\left(x^{*}\right)$ yield an evolutionary repellor. If $x^{*}$ is chosen such that the opposite of inequality (6) holds, then $e\left(x^{*}\right)>b_{c r i t}^{\prime \prime}\left(x^{*}\right)$, and trade-offs with an intermediate curvature (i.e., with $b_{\text {crit }}^{\prime \prime}\left(x^{*}\right)<$ $b^{\prime \prime}\left(x^{*}\right)<e\left(x^{*}\right)$ ) lead to so-called Garden of Eden $(G o E)$ points (Geritz et al., 1998; Hofbauer and Sigmund, 1990). A Garden of Eden point is an evolutionarily stable singularity, i.e., if the population is exactly at this point, then it stays there forever; but it is not attracting, i.e., starting with a dispersal strategy near but not exactly at the Garden of Eden point, the population evolves away from it. 
(a)

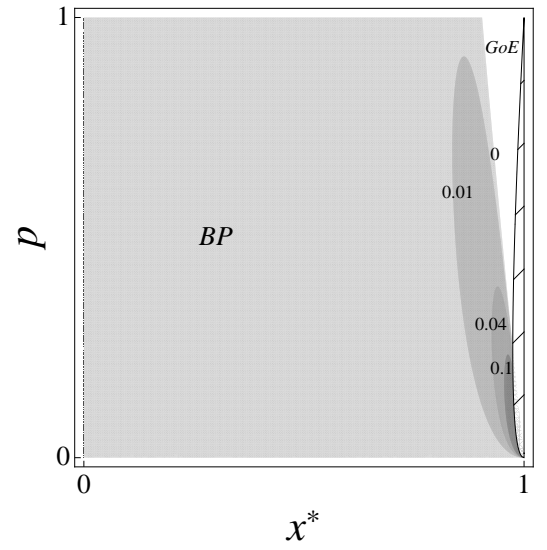

(b)

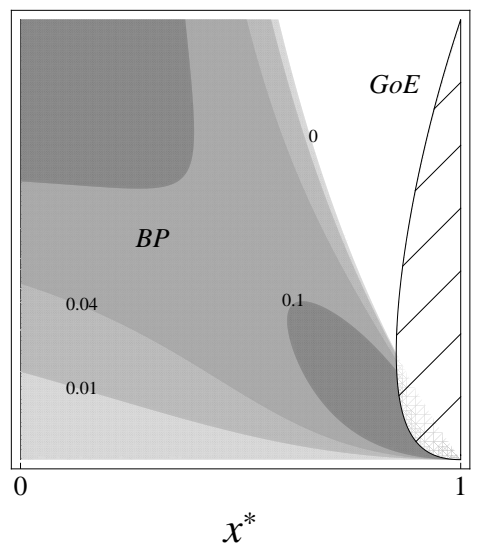

(c)

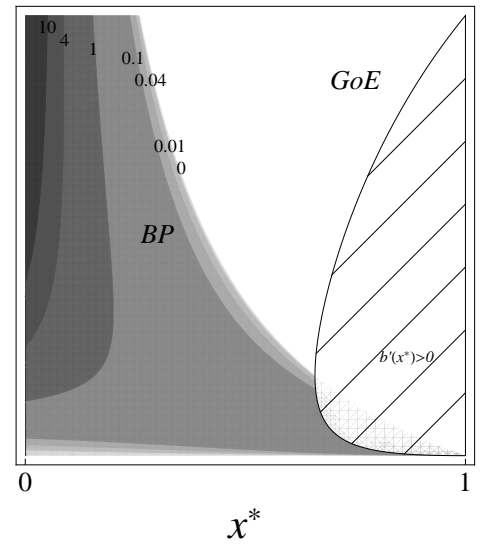

Figure 1: The grey regions show where $M<0$, i.e., inequality (6) is satisfied and where the critical function is decreasing. The shades of grey indicate the width of the interval $\left(b_{c r i t}^{\prime \prime}\left(x^{*}\right) / b\left(x^{*}\right), e\left(x^{*}\right) / b\left(x^{*}\right)\right)$, where evolutionary branching $(B P)$ occurs. In white regions $b_{c r i t}^{\prime \prime}\left(x^{*}\right) / b\left(x^{*}\right)<e\left(x^{*}\right) / b\left(x^{*}\right)$ holds and trade-offs of intermediate curvature make singular strategies Garden of Eden points $(G o E)$. In the dashed region, the critical function is increasing so that $x^{*}$ could be singular only with an increasing trade-off function, which is not of biological interest. Parameters: (a) $s=0.1$, (b) $s=0.5$, (c) $s=0.9$.

The grey regions of Fig. 1 show where inequality (6) is fulfilled and the critical function is decreasing; i.e., where evolutionary branching is possible for realistic trade-offs. The different shades of grey indicate the normalised range of curvature $b_{c r i t}^{\prime \prime}\left(x^{*}\right) / b\left(x^{*}\right)-e\left(x^{*}\right) / b\left(x^{*}\right)$, where the trade-off yields evolutionary branching. For low adult survival $s$, this range is narrow (Fig. 1a). Increased adult survival only slightly increases the range in which the trade-off must lie for branching to occur (Fig. 1b). High adult survival and medium to high survival during dispersal increase the range further, but diversification of dispersal strategies remains unlikely (Fig. 1c). Further evolutionary branching can be excluded, since there exist only two environmental feedbacks (see Section 2).

Note that sufficiently convex trade-offs $\left(b^{\prime \prime}\left(x^{*}\right)>b_{\text {crit }}^{\prime \prime}\left(x^{*}\right)\right)$ always yield evolutionary repellors. The boundary strategy $x=0$ is always repelling because of kin competition (see Appendix $\mathrm{C}$ ). The strategy on the upper boundary $x=1$ is also always repelling (see Appendix C). At the upper boundary kin competition vanishes and all sites are equal (whether offspring have been born on the site or not), which means no selection is favouring dispersal. Dispersal is however selected against both because a dispersed offspring has a chance of dying and because of the loss of fecundity through the dispersal-fecundity trade-off, so that $x=1$ is repelling. Because the boundary strategies are repelling, a repellor in the interior implies that at least two attracting singularities exist in $(0,1)$, one on each side of the interior repellor. Convex trade-offs therefore readily lead to alternative stable dispersal strategies. Concave trade-offs always lead to $C S S \mathrm{~s}$ since $b^{\prime \prime}\left(x^{*}\right)$ is negative and smaller than $e\left(x^{*}\right)$ and $b_{c r i t}^{\prime \prime}\left(x^{*}\right)$, which are both positive. 


\subsection{Effect of adult survival and survival during dispersal on dispersal strategy}

To study the effect of adult survival on the attracting singular strategy $x^{*}$, we implicitly differentiate the singularity condition $D\left(x^{*}\right)=\partial w /\left.\partial y\right|_{y=x=x^{*}}=0$ with respect to $s$. Rearranging terms leads to

$$
\frac{d x^{*}}{d s}=-\frac{\frac{\partial D}{\partial s}}{\frac{\partial D}{\partial x^{*}}} .
$$

For attracting singular strategies the denominator on the right hand side is always negative. Therefore, the numerator determines whether the singular strategy is increasing or decreasing with changing adult survival. In Appendix D.1 we show that $\frac{\partial D}{\partial s}<0$ and therefore the attracting dispersal strategy always decreases with adult survival (note that this holds also for the special case where we assume that $b(x)=b$ is constant, i.e., for the model without a trade-off). When more adults survive until reproduction, more patches are occupied, which selects for a decrease in dispersal, since it is harder to conquer new patches. Furthermore, more competitors arrive at a natal patch which increases competition locally with strangers and selects for less dispersal to increase the chance of retaining the natal patch (Gandon and Michalakis, 1999). In other words, kin competition becomes weaker when more immigrants dilute the non-dispersed siblings, and therefore dispersal decreases.

Next, we study the effect of survival during dispersal $p$ on the attracting singular strategy $x^{*}$. Analogously to Eq. (7), $x^{*}$ decreases with increasing $p$ if $\frac{\partial D}{\partial p}<0$. In Appendix D.2 we show that the attracting strategy decreases with $p$ if $\frac{p^{2} x^{* 2}}{\left(1-x^{*}\right)^{2}}<\frac{1-s}{s^{2}}$. This condition is the same as inequality (6). When the probability of survival during dispersal increases, the dispersal strategy can decrease, increase or vary non-monotonically. As $p$ increases, individuals' prospects to survive dispersal ameliorates, which selects for $x^{*}$ to increase with $p$; but more competitors arrive at the natal site, selecting for $x^{*}$ to decrease with $p$. Depending on the relative weight of these effects, one is dominating the other which makes all three scenarios possible (see the next section for examples). Without the trade-off (i.e., if $b$ is constant), the attracting dispersal strategy is always a non-monotone function of $p$ for $s<1$ (see Appendix D.2), whereas for $s=1$, we recover the result $x^{*}=1 /(2-p)$ of Hamilton and May (1977), which is an increasing function of $p$.

\section{Example}

In this section we illustrate our general results with a numerical example. We choose the tradeoff function $b(x)=\alpha x^{3}+\beta x^{2}+\gamma x+\delta$ with parameters such that the trade-off function is $(i)$ decreasing, (ii) non-negative and with $s=0.8$ and $p=0.2$ it is (iii) tangential to a critical function at $x^{*}=0.5$ with $(i v)$ intermediate convexity such that $e\left(x^{*}\right)<b^{\prime \prime}\left(x^{*}\right)<b_{c r i t}^{\prime \prime}\left(x^{*}\right)$ (Fig. $2 \mathrm{a})$. Hence, $x^{*}$ is an evolutionary branching point. Note that for the chosen values of $s$ and $p$, branching is impossible in the grey region of Fig. 2a, because inequality (6) is violated. 
(a)

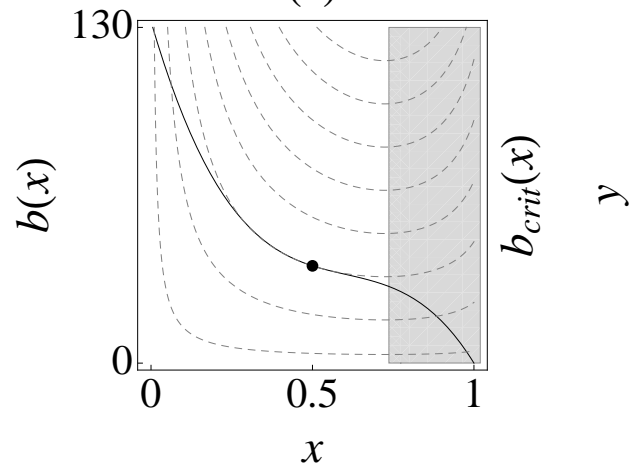

(b)

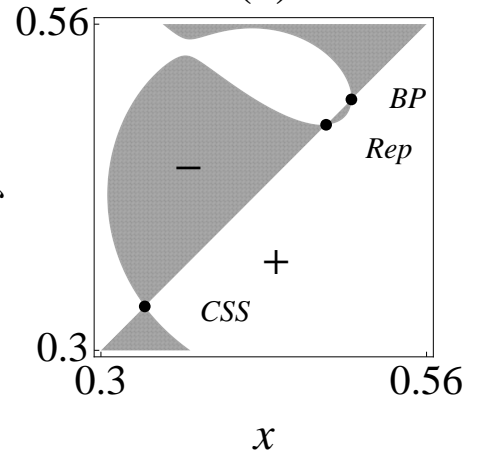

(c)

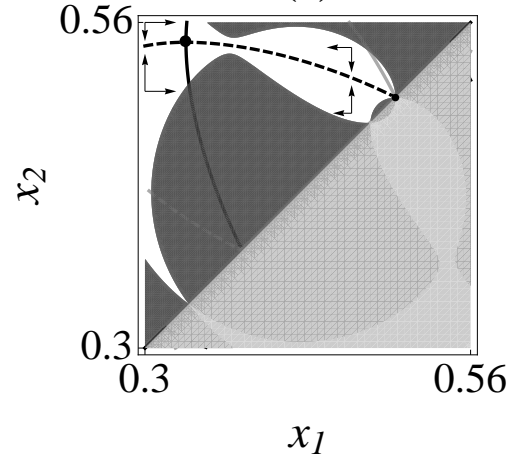

Figure 2: (a) The trade-off function $b(x)=-369.3 x^{3}+667.9 x^{2}-430.915 x+132.314$ (black) with a singular strategy at $x^{*}=0.5$ (dot). Dashed curves are critical functions for different initial values $b_{0}$. In the grey region, inequality (6) is violated so that evolutionary branching cannot occur with any trade-off (In this figure, the edge of the grey region seems to be at the minima of the critical functions, but this is a mere coincidence; see Fig. 1). Parameters: $s=0.8$, $p=0.2$. (b) The pairwise invasibility plot with parameters as given in (a). Abbreviations: $C S S$ : attracting ESS (also known as continuously stable strategy); Rep: repellor; BP: branching point. Parameters as in (a). (c) The mutual invasibility plot with arrows indicating the direction of dimorphic coevolution. Without loss of generality, we assume $x_{1}<x_{2}$, hence the lower half of this figure is greyed out. The lines within the white area (the area of coexistence) are isoclines at which the selection gradient is zero in either the $x_{1}$-direction or $x_{2}$-direction (dashed); colour indicates whether isoclines are evolutionarily stable (black) or not (grey). The intersection of lines correspond to the dimorphic evolutionary singularity. Parameters as in (a).

Fig. $2 \mathrm{~b}$ shows the pairwise invasibility plot for the above example, obtained as a sign plot of the logarithm of the invasion fitness in Eq. (2). There are three evolutionary singularities: an attracting and evolutionarily stable strategy $(C S S)$, an evolutionary repellor $(R e p)$, and the branching point $x^{*}(B P)$. The first two singularities correspond to points where the trade-off function is tangent to other critical functions not shown in Fig. 2a.

The adaptive dynamics after evolutionary branching is shown in Fig. 2c. Within the area of coexistence, the two resident dispersal strategies $x_{1}$ and $x_{2}$ evolve in the direction of their respective selection gradients (arrows). Evolution stops at the dimorphic singularity, the intersection of the isoclines of the selection gradients. The isoclines indicate the lines where the local fitness gradients vanish with respect to the $x_{1^{-}}$or $x_{2^{-}}$direction. We obtained the evolutionary isoclines by deriving the dimorphic invasion fitness $w\left(x_{1}, x_{2}, y\right)$ (Geritz et al., 1998). The dimorphic singularity is locally attracting (Leimar, 2009) and also evolutionarily stable. Since this model has two environmental feedback variables, branching into more than two strategies is impossible. Notice, however, that in the example of Fig. 2c, the evolutionary trajectory may easily leave the area of coexistence if $x_{1}$ (the strategy with lower dispersal) evolves faster than $x_{2}$. In this case, the strategy with higher dispersal goes extinct, and the remaining monomorphic population evolves to the $C S S$. Although the example in Fig. 2a,b,c demonstrates evolutionary branching, this appears to be an infrequent outcome. Fig. 3a shows the bifurcation diagram 
(a)

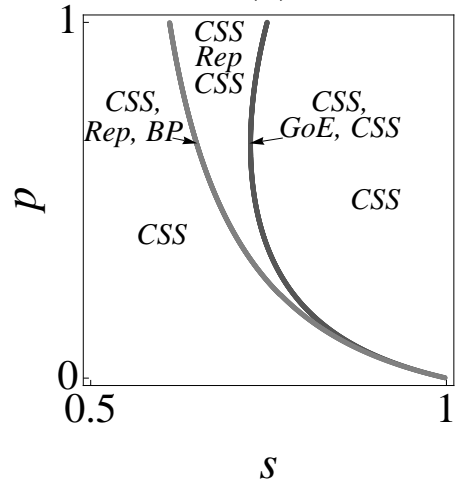

(b)

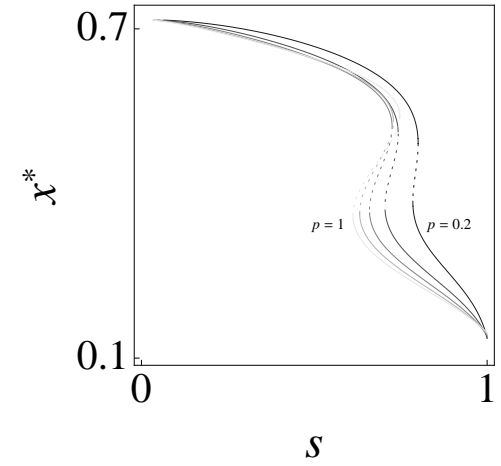

(c)

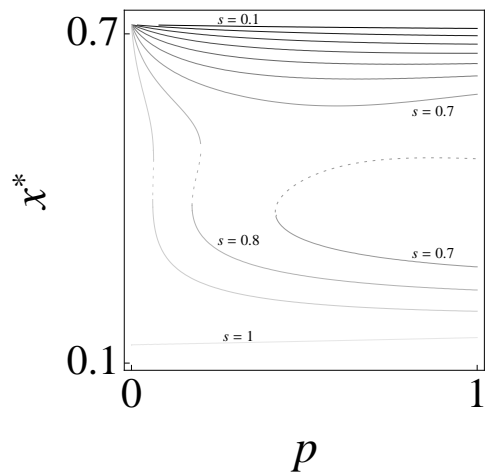

Figure 3: (a) The two parameter bifurcation diagram of evolutionary singularities assuming the trade-off function as given in Fig. 2a. Abbreviation: GoE: Garden of Eden (convergence unstable ESS). Note that the areas "CSS, Rep, BP" and "CSS, GoE, CSS" are exceedingly narrow. (b) \& (c) The bifurcation diagrams for $s$ and $p$, respectively. Dotted lines indicate repellors. At the upper bifurcation points GoEs exist and at the lower $B P \mathrm{~s}$; the attractors are $C S S$ s elsewhere. The lighter the lines, the higher the $p$ and $s$ value, respectively ( $p$ varies between 0.2 and 1 with steps of $0.2 ; s$ varies between 0.1 and 1 with steps of 0.1 ).

of evolutionary singularities under the trade-off in Fig. 2a, obtained by solving $D\left(x^{*}\right)=0$ and evaluating the stability criteria in (4) and (5) numerically. For most values of $s$ and $p$, the model exhibits a single convergence and evolutionarily stable dispersal strategy $(C S S)$, but there is also a sizeable area of the parameter space with two $C S S$ s separated by a repellor. In the latter area evolution leads to either of the two $C S S$ s depending on the initial trait value. The light grey area of Fig. 3a is a very narrow region where one singularity is an evolutionary branching point. In a similarly narrow region (dark grey in Fig. 3a), the repellor between the two CSSs is a Garden of Eden strategy, i.e., a repellor that is evolutionarily stable. Fig. 3b shows that the attracting singularity always decreases with increasing adult survival, $s$ (as proven in the previous section). In accordance with Fig. 3a, there may be alternative attractors of dispersal evolution when adult survival is relatively high. Fig. 3c demonstrates that the attracting dispersal strategy may increase or decrease with the probability of survival during dispersal $(p)$, and in this example dispersal is monotonically decreasing over the entire range $0<p<1$ when adult survival is either low or high but still sufficiently smaller than 1 . In Fig. 3b,c the dotted lines indicate repellors. Where a repellor collides with an evolutionary attractor, i.e., at fold bifurcation points, slight changes in the survival probabilities lead to an evolutionary catastrophe, whereby the former attractor disappears and the population evolves to another attractor at a very different dispersal probability.

\section{Discussion}

We have investigated the evolution of dispersal in an extended version of the Hamilton-May model (Hamilton and May, 1977), with a fecundity-dispersal trade-off and with mortality be- 
tween competition and reproduction (i.e., a factor of adult survival in the semelparous life cycle). Here dispersal helps offspring to avoid kin competition and potentially escape to a site with fewer competitors (i.e., where there was no surviving parent and thus only immigrants compete), whereas the costs are reduced fecundity and a chance of mortality during dispersal. We have found that in this extended model, all generic types of evolutionary singularities can occur, including evolutionary branching and evolutionary repellors; the latter leads to multiple attractors of dispersal evolution. In contrast, when there is no trade-off (i.e., fecundity is constant), there is always a single attractor which is evolutionarily stable (Appendix A).

Evolutionary branching requires multiple dispersal strategies to coexist near an evolutionary singularity. Such coexistence is impossible in the Hamilton-May model, but becomes possible with adult mortality (see inequality (6)). Many metapopulation models of dispersal evolution assume that catastrophes may extinguish local populations (e.g., Comins et al., 1980; Gandon and Michalakis, 1999; Olivieri et al., 1995; Parvinen et al., 2003; Ronce et al., 2000a,b). Adult survival in our model is analogous to surviving such a catastrophe, where the local "population" that survives the catastrophe is a single individual. Olivieri et al. (1995) showed that two dispersal strategies can coexist in a metapopulation model with catastrophes, although in their model the singular dispersal strategies are always ESSs.

When coexistence near an evolutionary singularity is possible, then the shape of the tradeoff function determines whether evolutionary branching occurs. The importance of the tradeoff shape was highlighted by Ronce and Olivieri (1997), who found an evolutionarily stable dimorphism in fecundity when it stands in a trade-off with parental survival according to a convex-concave trade-off function (similar to our example in Fig. 2) in a population with overlapping generations. Dispersal was linked to reproduction and branching in fecundity amounted to branching in dispersal, resulting in a strategy with a high fecundity, high dispersal and low parental survival and a strategy characterised by low fecundity, low dispersal and high parental survival. Many other models, however, assume only a fixed mortality cost of dispersal, which amounts to a linear trade-off between the number of dispersed and non-dispersed offspring; this rigid trade-off explains, for example, why evolutionary branching does not occur in the model of Olivieri et al. (1995). When the trade-off is flexible, as in case of a fecundity-dispersal trade-off, then given coexistence, one can always find trade-off functions that yield evolutionary branching (Kisdi, 2006, 2014). Whether these trade-offs are biologically realistic can be evaluated by empirical studies. In Fig. 3a, we show that any evolutionary outcome is possible in the present model with a decreasing trade-off function, which is a minimal requirement for biological realism when the trade-off derives from resource allocation between dispersal-related investments and fecundity.

Two previous models have been used to study the evolution of dispersal with arbitrary shapes of trade-offs. Cohen and Motro (1989) assumed a trade-off between dispersal and fecundity as in our model, but assumed $100 \%$ adult survival, such that coexistence was impossible in their model. They found that there is always at least one attracting singularity, and since dispersal implies a cost in fecundity, all singular dispersal strategies must be lower than the Hamilton-May solution. Note that this does not hold when there is adult mortality, since the empty sites generate an extra selective force promoting dispersal. Levin and Muller-Landau (2000) investigated the evolution of seed size, assuming that small seeds are more likely to disperse but are competitively inferior to large seeds. Varying seed size thus generates a trade-off between dispersal and potency, a combined measure of the number of seeds and their com- 
petitive weight. Four types of evolutionary singularities without coexistence can occur in this model (including the Garden of Eden scenario), but since $100 \%$ adult survival is assumed, coexistence and evolutionary branching is impossible. Asymmetric competition is known to facilitate evolutionary branching of seed size when some sites are empty before dispersal (Geritz et al., 1999).

Evolutionary branching of dispersal has been found in a number of models, but has not been linked to the shapes of trade-offs involved. Most of these models assume no trade-off (Doebeli and Ruxton, 1997; Mathias et al., 2001) or only the linear trade-off implied by a fixed mortality cost of dispersal (Massol et al., 2011; Parvinen, 1999, 2002; Parvinen and Metz, 2008). The simulation study of Fronhofer et al. (2011) found evolutionary branching in sexually reproducing populations under a fixed trade-off between fecundity and survival during dispersal in a model where temporal variability selects for dispersal. With the exceptions of Parvinen and Metz (2008) and Massol et al. (2011), models with evolutionary branching of dispersal also assumed large local populations where kin competition does not play a role.

The multiple attractors of dispersal evolution predicted by our model are intimately linked to the shape of the underlying trade-offs. One numerical example for multiple attractors was found by Levin and Muller-Landau (2000), when they assumed an ad hoc non-monotone relationship between dispersal and potency (the total competitive weight represented by the offspring). In our model, potency is given by $b(x)[1-(1-p) x]$, which is a monotone decreasing function of $x$ whenever $b(x)$ is non-increasing (note, however, Levin and Muller-Landau (2000) assumed that small seeds disperse better; in their model $b(x)$ is an increasing function of $x$ ). Our model demonstrates that the existence of an evolutionary repellor, and hence of multiple attractors, depends not on the monotonicity but on the curvature of the trade-off: when the trade-off is more convex than the critical function at the point of tangent, the singularity is a repellor (Fig. 2a; de Mazancourt and Dieckmann 2004).

A fundamentally different mechanism leads to multiple attractors in the model of Billiard and Lenormand (2005). They assume genetic polymorphism in a locus involved in local adaptation to contrasting habitats in a heterogeneous landscape. When dispersal is low, the locally favoured allele is frequent in each local population, which selects against immigrants carrying the "wrong" allele and thereby stabilises low dispersal. In contrast, high dispersal homogenises the allele frequencies and thus reduces the disadvantage of dispersal, so that kin competition can maintain high dispersal. Also the joint adaptive dynamics of dispersal and a local adaptation trait readily leads to multiple attractors, but here selective forces on the trait under local adaptation play a major role (Kisdi, 2002; Nurmi and Parvinen, 2011).

Multiple attractors can also arise such that one attractor is an evolutionarily stable dispersal strategy and another is an evolutionarily stable dispersal dimorphism. The simultaneous existence of a monomorphic ESS and a dimorphic ESS is a generic feature of adaptive dynamics (Geritz et al., 1999). In our example, the region where a dimorphic ESS exists is small (see Fig. 5 in Appendix E), yet somewhat larger than the region where evolutionary branching occurs (Fig. 3a). In the part where a dimorphic ESS exists but branching does not occur, a population can evolve to the dimorphic ESS only if it starts dimorphic (e.g., by immigration).

In our model, the attracting dispersal strategy always decreases with adult survival. This is different from how dispersal changes with the catastrophe rate in structured metapopulations (Parvinen et al., 2003; Ronce et al., 2000a,b), but agrees with metapopulation models that 
assume saturated local populations (Comins et al., 1980; Olivieri et al., 1995). Our model is analogous to the latter case since a site is saturated with one individual.

Contrastingly, the attracting dispersal strategy of our model may either increase or decrease with increasing survival during dispersal. Higher survival selects for dispersal as dispersal is then safer, but also selects against dispersal because more immigrants arrive at the natal site of the offspring and therefore more offspring need to stay in the natal patch in order to protect the site against competitors. These conflicting forces can lead to a non-monotonic relationship between dispersal and survival during dispersal (Comins et al., 1980; Gandon, 1999; Gandon and Michalakis, 1999; Heino and Hanski, 2001; Kisdi, 2004). Interestingly, in the example of Fig. 2, we also find a monotone decreasing relationship, i.e., here increasing the probability of survival during dispersal leads to even lower (and not higher) dispersal, which is a novel pattern in dispersal evolution.

Close to fold bifurcation points, i.e., at points where an attractor disappears via colliding with a repellor, slight changes in the probability of adult survival or in survival during dispersal may cause major shifts in the evolved dispersal strategies (see Figs 2e,f). At such points, 'evolutionary catastrophes' occur and the population evolves rapidly to another attractor (Rand and Wilson, 1993).

The present results highlight the importance of the shape of trade-off in generating disruptive selection and hence polymorphisms or multiple attractors in dispersal evolution. However, we have made just one step in exploring the rich trade-off structure that determines the evolution of dispersal (Bonte et al., 2012; Travis et al., 2012). Most importantly, models should go beyond considering a single trade-off linking only two life-history parameters, and incorporate multiple trade-offs between interacting traits.

In the present model, we assumed that the probability of dispersal is a purely genetically determined trait. Even though dispersal is indeed a heritable trait (see Ronce, 2007; birds: Doligez et al., 2009; Hansson et al., 2003; plants: Clay, 1982; Venable and Burquez, 1989), in reality other factors such as the quality of the natal habitat, local population density, the strength of kin competition, and the competitive ability of the individual also influence dispersal (Benard and McCauley, 2008; Clobert et al., 2009). Function-valued dispersal strategies have been investigated in the context of density-dependent dispersal (e.g., Gyllenberg and Metz 2001; Parvinen et al. 2012), kin competition (Ezoe and Iwasa, 1997; Kisdi, 2004; Ronce et al., 1998), and dispersal in relation to body condition (Gyllenberg et al., 2011a,b; Kisdi et al., 2012), but these models make only the simplest assumptions about the costs of dispersal. Integrating improved modelling of dispersal-related trade-offs with plastic dispersal strategies is an important step in exploring the role of plasticity and genetic diversification in the evolution of dispersal.

\section{Acknowledgments}

This research was funded by the Finnish Graduate School in Computational Sciences (FICS) and by the Academy of Finland. We are grateful to Kalle Parvinen and an anonymous reviewer for helpful suggestions. We thank Matthew Osmond for comments on the manuscript. 


\section{Appendix}

\section{A Adaptive dynamics of dispersal without a trade-off}

In this section we analyse the model assuming no trade-off, i.e., assuming constant fecundity $(b(x)=b)$. The fitness function is then independent of fecundity and is given by:

$$
w(x, y)=s\left(\frac{(1-y)}{x p s+(1-y)}+\frac{y p s}{x p s+(1-x)}+\frac{y p(1-s)}{x p s}\right) .
$$

We derive the singular strategy by solving $D\left(x^{*}\right)=0$ and get:

$$
x^{*}=\frac{2-s(p(2-s)+2+\sqrt{p} \sqrt{4+s(p s-4)})}{2-2 s(p(2-p s)+1)} .
$$

When $s=1$, the singular strategy simplifies to $1 /(2-p)$, which has been obtained by Hamilton and May (1977). The singular strategy is evolutionarily stable if

$$
\left.\frac{\partial^{2} w(x, y)}{\partial y^{2}}\right|_{x=y=x^{*}}=-\frac{2 p s^{2} x^{*}}{\left(1-x^{*}+p s x^{*}\right)^{3}}<0
$$

which is always true. The singular strategy is convergence stable if

$$
\frac{\partial^{2} w(x, y)}{\partial y^{2}}+\left.\frac{\partial^{2} w(x, y)}{\partial x \partial y}\right|_{x=y=x^{*}}=-\frac{1-s}{x^{*^{2}}}-\frac{p s^{2}\left(p s\left(1-x^{*}\right)+x^{*}\left(2(1-p s)+p^{2} s^{2}\right)\right)}{\left(1-x^{*}+p s x^{*}\right)^{3}}<0,
$$

which is always true and therefore the singular strategy is a $C S S$ when the trade-off function is constant.

\section{B Critical functions and curvatures}

\section{B.1 Imaginary part of ArcTanh}

When the argument of ArcTanh lies outside of $(-1,1)$ the function value is complex. If $z \in \mathbb{R}$ with $z^{2}>1$, then we can write

$$
\operatorname{ArcTanh}(z)=\alpha+i \beta
$$

where $\alpha=1 / 2 \operatorname{Ln}[(z+1) /(z-1)]$ and $\beta= \pm \pi / 2$. Note that the imaginary part of the ArcTanh is positive if $z<-1$ and negative if $z>1$. The $\operatorname{argument}$ of $\operatorname{ArcTanh}(z)$ is

$$
z=\frac{x\left[(1-p s)^{2}-s\right]-[(1-p s)-s]}{p \sqrt{s^{3}}},
$$

which is linear in $x$. Let us look at the values of $z$ when $x=0$ and $x=1$. When $x$ is at the lower boundary of trait space $z$ simplifies to

$$
\left.z\right|_{x=0}=\frac{s(1+p)-1}{p s^{3 / 2}}
$$


and when $x=1, z$ is

$$
\left.z\right|_{x=1}=\frac{p s-1}{\sqrt{s}} .
$$

The righthand sides of Eq. (10) and Eq. (11) are always less than 1 . If $p>1 / s-\sqrt{1 / s}$, then both (10) and (11) are greater than -1 , so that the ArcTanh function evaluates to real numbers for all $x$. If the reverse inequality holds, then both (10) and (11) are less than -1 , so that the ArcTanh function evaluates to a complex number with imaginary part $\beta=\pi / 2$. Therefore when $f(x)$ is complex, then $f\left(x_{0}\right)$ is also complex and the imaginary parts cancel in $f\left(x_{0}\right)-f(x)$.

\section{B.2 Curvature of the critical function}

Here we investigate the curvature of the critical function $b_{\text {crit }}\left(x^{*}\right)$. We derive the second derivative and get

$$
b_{\text {crit }}^{\prime \prime}(x)=\frac{b_{\text {crit }}(x)}{d(p, s, x)^{2} x^{2}}\left[2(1-s)^{2}(1-x)^{4}+2 p s(1-s) x(1-x) A+p^{2} s^{2} x^{2}\left(4(1-x)^{2}(1-s)+B\right)\right],
$$

where

$$
d(p, s, x)=p^{2} s^{2} x^{2}+(1-s)(1-x)^{2}+2 s p x(1-x),
$$

which is positive. The term in square brackets on the right hand side of Eq. (12) has three terms, of which the first one is positive. The second term is positive whenever

$$
A=\left(4(1-x)^{2}-s(1-2 x)\right)>0 .
$$

This always holds when $x>1 / 2$. If $x<1 / 2$, then $A$ is decreasing in $s$. When taking the worst case $s=1$, inequality (14) simplifies to $4 x^{2}-6 x+3$, which is positive for all $x$. Hence, also the second term of Eq. (12) is positive. The last term of Eq. (12) is positive if $B$ is positive, where

$$
B=8-6 s+s^{2}+C
$$

and

$$
C=2 x(2-p s)((2-p s) x-4+2 s) .
$$

Only $C$ depends on $x$, in particular it is quadratic in $x$. The coefficient of the quadratic term of Eq. (15) is positive. The minimum value of $C$ is reached when $x=(2-s) /(2-p s)$. Substituting the minimum into $B$ gives $(2-s) s>0$, which is positive. Therefore Eq. (12) is positive and hence $b_{\text {crit }}(x)$ is convex.

\section{B.3 Critical curvature for evolutionary stability}

Here we show that $e\left(x^{*}\right)$, defined as the minimal value of $b^{\prime \prime}\left(x^{*}\right)$ that violates the evolutionary stability condition in Eq. (4) for the singularity $x^{*}$, is always positive. From its definition, we obtain

$$
e\left(x^{*}\right)=\left(\frac{d\left(p, s, x^{*}\right)-p s^{2} x^{*}}{x^{*} d\left(p, s, x^{*}\right)}\right)^{2}+\frac{p s^{2}\left[x^{*}-1+s\left(1-x^{*}(1+p)\right)\right]^{2}\left(1-x^{*}+p s x^{*}\right)}{x^{*} d\left(p, s, x^{*}\right)^{3}},
$$


where $d(p, s, x)$ is given by Eq. (13). Since the first term of Eq. (16) is strictly non-negative and the second term is positive, $e\left(x^{*}\right)$ is positive.

\section{Stability on the boundary}

The strategy located at the lower boundary of the trait space, $x=0$, is repelling if $D(0)>0$, which is equivalent to $1-s>0$. The strategy located at the upper boundary of the trait space, $x=1$, is repelling if

$$
D(1)<0 \quad \Leftrightarrow \quad 1-\frac{1}{p}+\frac{b^{\prime}(1)}{b(1)}<0
$$

which is also true for decreasing trade-offs.

\section{The effect of $s \& p$ on the attracting singular strategy}

\section{D.1 The effect of $s$ on the attracting singular strategy}

Here we investigate how the attracting singular strategy changes with increased adult survival. From Eq. (7) we have

$$
\frac{\partial D}{\partial s}=-\frac{p s\left(1-x^{*}\right)\left[2 p s x^{*}+\left(1-x^{*}\right)(2-s)\right]}{d\left(p, s, x^{*}\right)\left(1-x^{*}+p s x^{*}\right)^{2}} .
$$

Since $d\left(p, s, x^{*}\right)$ as given in Eq. (13) is positive, the right-hand side of Eq. (17) is negative for attracting singular strategies. Hence Eq. (7) is negative and dispersal decreases with survival.

Next consider the case when the trade-off function is constant. In this case we can explicitly derive the singular strategy (see Eq. (9)). The singular strategy increases with adult survival if

$$
\frac{d x^{*}}{d s}=\frac{\sqrt{p}\left(6 s(1-p s)+4\left(p^{2} s^{2}-1\right)+s\left(s^{2} p-2\right)\right)}{2 \sqrt{4+s(p s-4)}(1+s(p(p s-2)-1))^{2}}+\frac{p\left((2-s) s+2(1-p s)^{2}\right)}{2(1+s(p(p s-2)-1))^{2}}<0 .
$$

With the command Reduce of Mathematica, we prove that inequality (18) is always fulfilled. Hence, the singular strategy decreases with increasing adult survival.

\section{D.2 The effect of $p$ on the attracting singular strategy}

As shown in the main text, the sign of $d x^{*} / d p$ coincides with the sign of

$$
\frac{\partial D}{\partial p}=\frac{s^{2}\left(p^{2} s^{2} x^{* 2}-(1-s)\left(1-x^{*}\right)^{2}\right)}{d\left(p, s, x^{*}\right)\left(1-x^{*}+p s x^{*}\right)^{2}} .
$$

We can see that the denominator is always positive. Therefore Eq. (19) is negative if $p^{2} s^{2} x^{* 2}-$ $(1-s)\left(1-x^{*}\right)^{2}<0$, which is equivalent to

$$
\frac{p^{2} x^{* 2}}{\left(1-x^{*}\right)^{2}}<\frac{1-s}{s^{2}}
$$


In particular, when $s=1$ then Eq. (20) simplifies to $p^{2} x^{* 2}<0$ which is never satisfied. Hence, the attracting singularity increases with $p$ when all adults survive until reproduction. When inequality (20) is fulfilled dispersal decreases with increasing survival during dispersal.

Next, we analyse the change of the singular strategy as survival during dispersal increases in the case where the trade-off function is constant. The singular strategy as given in Eq. (9) decreases with increasing $p$ if

$$
\frac{d x^{*}}{d p}=\frac{s\left(s-2-\frac{p s^{2}+4+s(p s-4)}{2 \sqrt{p} \sqrt{4+s(p s-4)}}\right)}{2(1-s)-2 p s(p s-2)}-\frac{s(p s-1)(2-s(2-p(s-2)+\sqrt{p} \sqrt{4+s(p s-4)}))}{(1+s(p(p s-2)-1))^{2}}<0 .
$$

Instead of analysing the sign change of Eq. (21), we show in Fig. 4 that the $d x^{*} / d p$ changes sign for all $s<1$. Then, we can conclude that the singular strategy changes non-monotonically for all $s<1$.

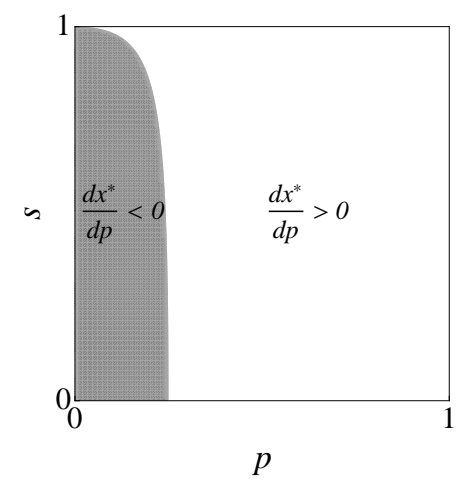

Figure 4: A sign plot of $\frac{d x^{*}}{d p}$ when the trade-off function is constant. 


\section{E Dimorphic ESS}

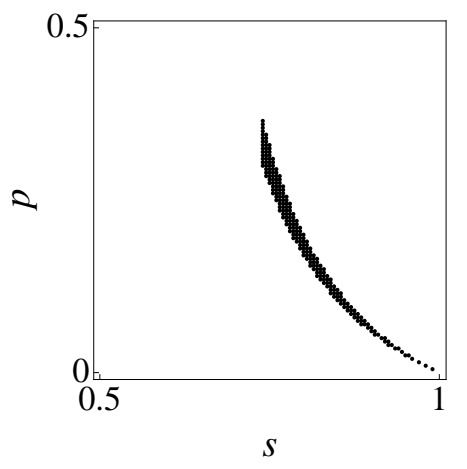

Figure 5: The black region indicates the existence of a dimorphic ESS assuming the tradeoff function as in Fig.2. We numerically obtained the dimorphic ESS by setting the selection gradients $\left.\frac{\partial w\left(x_{1}, x_{2}, y\right)}{\partial y}\right|_{y=x_{1}}$ and $\left.\frac{\partial w\left(x_{1}, x_{2}, y\right)}{\partial y}\right|_{y=x_{2}}$ equal to zero. We then evaluated the stability criteria (analog to (4)) at the singular strategy numerically and checked if the equilibrium population densities were positive at that point.

\section{References}

Benard, M. F., McCauley, S. J., 2008. Integrating across life-history stages: consequences of natal habitat effects on dispersal. The American naturalist 171 (5), 553-67.

Billiard, S., Lenormand, T., 2005. Evolution of migration under kin selection and local adaptation. Evolution 59 (1), 13-23.

Bonte, D., Hovestadt, T., Poethke, H.-J., 2010. Evolution of dispersal polymorphism and local adaptation of dispersal distance in spatially structured landscapes. Oikos 119 (3), 560-566.

Bonte, D., Van Dyck, H., Bullock, J. M., Coulon, A., Delgado, M. M., Gibbs, M., Lehouck, V., Matthysen, E., Mustin, K., Saastamoinen, M., Schtickzelle, N., Stevens, V. M., Vandewoestijne, S., Baguette, M., Bartoń, K. A., Benton, T. G., Chaput-Bardy, A., Clobert, J., Dytham, C., Hovestadt, T., Meier, C. M., Palmer, S. C. F., Turlure, C., Travis, J. M. J., 2012. Costs of dispersal. Biological Reviews 87, 290-312.

Burton, O. J., Phillips, B. L., Travis, J. M. J., 2010. Trade-offs and the evolution of life-histories during range expansion. Ecology Letters 13, 1210-1220.

Christiansen, F., 1991. On Conditions for Evolutionary Stability for a Continuously Varying Character. The American Naturalist 138 (1), 37-50.

Clay, K., 1982. Environmental and Genetic Determinants of Cleistogamy in a Natural Population of the Grass Danthonia spicata. Evolution 36 (4), 734-741. 
Clobert, J., Le Galliard, J.-F., Cote, J., Meylan, S., Massot, M., 2009. Informed dispersal, heterogeneity in animal dispersal syndromes and the dynamics of spatially structured populations. Ecology Letters 12 (3), 197-209.

Cohen, D., Motro, U., 1989. More on Optimal Rates of Dispersal : Taking into Account the Cost of the Dispersal Mechanism. The American Naturalist 134 (4), 659-663.

Comins, H. N., Hamilton, W., May, R. M., 1980. Evolutionarily stable dispersal strategies. Journal of Theoretical Biology 82 (2), 205-30.

de Mazancourt, C., Dieckmann, U., 2004. Trade-Off Geometries and Frequency-dependent Selection. The American Naturalist 164 (6), 765-778.

Doebeli, M., Ruxton, G. D., 1997. Evolution of dispersal rates in metapopulation models: branching and cyclic dynamics in phenotype space. Evolution 51 (6), 1730-1741.

Doligez, B., Gustafsson, L., Part, T., 2009. 'Heritability' of dispersal propensity in a patchy population. Proceedings of the Royal Society B: Biological Sciences 276, 2829-2836.

Eshel, I., 1983. Evolutionary and Continuous Stability. Journal of Theoretical Biology 103, 99-111.

Ezoe, H., 1998. Optimal dispersal range and seed size in a stable environment. Journal of Theoretical Biology 190 (3), 287-93.

Ezoe, H., Iwasa, Y., 1997. Evolution of Condition Dependent Dispersal: A Genetic Algorithm Search for the ESS Reaction Norm. Researches on Population Ecology 39 (2), 127-137.

Fronhofer, E. A., Kubisch, A., Hovestadt, T., Poethke, H.-J., 2011. Assortative mating counteracts the evolution of dispersal polymorphisms. Evolution 65 (9), 2461-9.

Gandon, S., 1999. Kin competition, the cost of inbreeding and the evolution of dispersal. Journal of Theoretical Biology 200 (4), 345-64.

Gandon, S., Michalakis, Y., 1999. Evolutionarily stable dispersal rate in a metapopulation with extinctions and kin competition. Journal of Theoretical Biology 199 (3), 275-90.

Geritz, S. A. H., Kisdi, É., Meszéna, G., Metz, J. A. J., 1998. Evolutionarily singular strategies and the adaptive growth and branching of the evolutionary tree. Evolutionary Ecology 12 (1), $35-57$.

Geritz, S. A. H., Kisdi, É., Yan, P., 2007. Evolutionary branching and long-term coexistence of cycling predators: critical function analysis. Theoretical Population Biology 71 (4), 424-35.

Geritz, S. A. H., Metz, J. A. J., Kisdi, É., Meszéna, G., 1997. Dynamics of Adaptation and Evolutionary Branching. Physical Review Letters 78 (10), 2024-2027.

Geritz, S. A. H., van der Meijden, E., Metz, J. A. J., 1999. Evolutionary dynamics of seed size and seedling competitive ability. Theoretical Population Biology 55 (3), 324-43. 
Gu, H., Danthanarayana, W., 1992. Quantitative genetic analysis of dispersal in Epiphyas postvittana. II . Genetic covariations between flight capacity and life-history traits. Heredity 68 (February 1991), 61-69.

Gu, H., Hughes, J., Dorn, S., 2006. Trade-off between mobility and fitness in Cydia pomonella L.(Lepidoptera: Tortricidae). Ecological Entomology 31, 68-74.

Gyllenberg, M., Kisdi, É., Utz, M., 2011a. Body condition dependent dispersal in a heterogeneous environment. Theoretical Population Biology 79 (4), 139-54.

Gyllenberg, M., Kisdi, É., Utz, M., 2011b. Variability within families and the evolution of bodycondition-dependent dispersal. Journal of Biological Dynamics 5 (2), 191-211.

Gyllenberg, M., Metz, J. A. J., 2001. On fitness in structured metapopulations. Journal of Mathematical Biology 268 (6), 545-560.

Hamilton, W., May, R. M., 1977. Dispersal in Stable Habitats. Nature 269, 578-581.

Hanski, I., Saastamoinen, M., Ovaskainen, O., 2006. Dispersal-related life-history trade-offs in a butterfly metapopulation. Journal of Animal Ecology 75 (1), 91-100.

Hansson, B., Bensch, S., Hasselquist, D., 2003. Heritability of dispersal in the great reed warbler. Ecology Letters 6, 290-294.

Heino, M., Hanski, I., 2001. Evolution of migration rate in a spatially realistic metapopulation model. The American Naturalist 157 (5), 495-511.

Hofbauer, J., Sigmund, K., 1990. Adaptive Dynamics and Evolutionary Stability. Applied Mathematics Letters 3 (4), 75-79.

Hughes, C. L., Hill, J. K., Dytham, C., 2003. Evolutionary trade-offs between reproduction and dispersal in populations at expanding range boundaries. Proceedings of the Royal Society B: Biological Sciences 270 Suppl, S147-50.

Karlsson, B., Johansson, A., 2008. Seasonal polyphenism and developmental trade-offs between flight ability and egg laying in a pierid butterfly. Proceedings of the Royal Society B: Biological Sciences 275 (1647), 2131-6.

King, E. G., Roff, D. A., 2010. Modeling the evolution of phenotypic plasticity in resource allocation in wing-dimorphic insects. The American Naturalist 175 (6), 702-16.

Kisdi, É., 2002. Dispersal: risk spreading versus local adaptation. The American Naturalist 159 (6), 579-596.

Kisdi, É., 2004. Conditional dispersal under kin competition: extension of the Hamilton-May model to brood size-dependent dispersal. Theoretical Population Biology 66 (4), 369-380.

Kisdi, É., 2006. Trade-off geometries and the adaptive dynamics of two co-evolving species. Evolutionary Ecology Research 8 (6), 959-973.

Kisdi, É., 2014. Construction of multiple trade-offs to obtain arbitrary singularities of adaptive dynamics. Journal of mathematical biology 70 (5), 1093-1117. 
Kisdi, É., Utz, M., Gyllenberg, M., 2012. CHAPTER 11- Evolution of condition-dependent dispersal. In: Clobert, J., Baguette, M., Benton, T. G., Bullock, J. M. (Eds.), Dispersal Ecology and Evolution. Oxford University Press, Oxford, Ch. 11, pp. 139-151.

Leimar, O., 2009. Multidimensional convergence stability. Evolutionary Ecology, 191-208.

Levin, S. A., Muller-Landau, H. C., 2000. The evolution of dispersal and seed size in plant communities. Evolutionary Ecology Research 2, 409-435.

Massol, F., Duputié, A., David, P., Jarne, P., 2011. Asymmetric patch size distribution leads to disruptive selection on dispersal. Evolution 65 (2), 490-500.

Mathias, A., Kisdi, É., Olivieri, I., 2001. Divergent evolution of dispersal in a heterogeneous landscape. Evolution 55 (2), 246-259.

Mole, S., Zera, A., 1993. Differential allocation of resouces underlies the dispersal-reproduction trade-off in the wing-dimorphic cricket, Gryllus rubens. Oecologia 93, 121-127.

Nurmi, T., Parvinen, K., 2011. Joint evolution of specialization and dispersal in structured metapopulations. Journal of Theoretical Biology 275 (1), 78-92.

Olivieri, I., Michalakis, Y., Gouyon, P.-H., 1995. Metapopulation Genetics and the Evolution of Dispersal. The American Naturalist 146 (2), 202-228.

Parvinen, K., 1999. Evolution of Migration in a Metapopulation. Bulletin of Mathematical Biology 61 (3), 531-550.

Parvinen, K., 2002. Evolutionary branching of dispersal strategies in structured metapopulations. Journal of Mathematical Biology 45, 106-124.

Parvinen, K., Dieckmann, U., Gyllenberg, M., Metz, J. A. J., jan 2003. Evolution of dispersal in metapopulations with local density dependence and demographic stochasticity. Journal of Evolutionary Biology 16 (1), 143-53.

Parvinen, K., Metz, J. A. J., 2008. A novel fitness proxy in structured locally finite metapopulations with diploid genetics, with an application to dispersal evolution. Theoretical Population Biology 73 (4), 517-28.

Parvinen, K., Seppänen, A., Nagy, J. D., 2012. Evolution of complex density-dependent dispersal strategies. Bulletin of Mathematical Biology 74 (11), 2622-2649.

Poethke, H.-J., Gros, A., Hovestadt, T., 2011. The ability of individuals to assess population density influences the evolution of emigration propensity and dispersal distance. Journal of Theoretical Biology 282 (1), 93-99.

Rand, D. A., Wilson, H. B., 1993. Evolutionary Catastrophes, Punctuated Equilibria and Gradualism in Ecosystem Evolution. Proceedings of the Royal Society B: Biological Sciences 253 (1337), 137-141.

Roff, A., Fairbairn, J., 1991. Wing Dimorphisms and the Evolution of Migratory Polymorphisms among the Insecta. American Zoologist 31 (1), 243-251. 
Roff, D. A., 1977. Dispersal in Dipterans: Its Costs and Consequences. The Journal of Animal Ecology 46 (2), 443-456.

Roff, D. A., 1984. The cost of being able to fly: A Study of Wing Polymorphism in two species of crickets. Oecologia 63 (1), 30-37.

Roff, D. A., 1986. The Evolution of Wing Dimorphism in Insects. Evolution 40 (5), 1009-1020.

Ronce, O., 2007. How Does It Feel to Be Like a Rolling Stone? Ten Questions About Dispersal Evolution. Annual Review of Ecology, Evolution, and Systematics 38 (1), 231-253.

Ronce, O., Clobert, J., Massot, M., 1998. Natal dispersal and senescence. Proceedings of the National Academy of Sciences of the United States of America 95 (2), 600-5.

Ronce, O., Olivieri, I., 1997. Evolution of Reproductive Effort in a Metapopulation with Local Extinctions and Ecological Succession. The American Naturalist 150 (2), 220-249.

Ronce, O., Perret, F., Olivieri, I., 2000a. Evolutionarily Stable Dispersal Rates Do Not Always Increase with Local Extinction Rates. The American Naturalist 155 (4), 485-496.

Ronce, O., Perret, F., Olivieri, I., 2000b. Landscape dynamics and evolution of colonizer syndromes: interactions between reproductive effort and dispersal in a metapopulation. Evolutionary Ecology 14, 233-260.

Rousset, F., Gandon, S., 2002. Evolution of the distribution of dispersal distance under distancedependent cost of dispersal. Journal of Evolutionary Biology 15 (4), 515-523.

Stirling, G., Fairbairn, D., Jensen, S., Roff, D. A., 2001. Does a negative genetic correlation between wing morph and early fecundity imply a functional constraint in Gryllus firmus? Evolutionary Ecology Research 3, 157-177.

Thomson, F. J., Moles, A. T., Auld, T. D., Kingsford, R. T., nov 2011. Seed dispersal distance is more strongly correlated with plant height than with seed mass. Journal of Ecology 99 (6), $1299-1307$.

Travis, J. M. J., Mustin, K., Bartoń, K. A., Benton, T. G., Clobert, J., Delgado, M. M., Dytham, C., Hovestadt, T., Palmer, S. C. F., Van Dyck, H., Bonte, D., 2012. Modelling dispersal: an eco-evolutionary framework incorporating emigration, movement, settlement behaviour and the multiple costs involved. Methods in Ecology and Evolution 3 (4), 628-641.

Venable, D., Burquez, A., 1989. Quantitative Genetics of Size, Shape , Life-History , and Fruit Characteristics of the Seed- Heteromorphic Composite Heterosperma pinnatum . I . Variation Within and Among Populations. Evolution 43 (1), 113-124.

Wratten, S. D., apr 1977. Reproductive strategy of winged and wingless morphs of the aphids Sitobion avenae and Metopolophium dirhodum. The Annals of applied Biology 85 (3), 319-31.

Zera, A., 1984. Differences in Survivorship, Development Rate and Fertility Between the Longwinged and Wingless Morphs of the Waterstrider, Limnoporus canaliculatus. Evolution 38 (5), $1023-1032$.

Zera, A., Denno, R. F., 1997. Physiology and ecology of dispersal polymorphism in insects. Annual Review of Entomology 42, 207-30. 